\title{
Observation on the Clinical Effect of Modified Circumcision in the Treatment of Phimosis and Excessive Foreskin
}

\author{
Jiechang Ju \\ Zhengzhou Central Hospital Affiliated to Zhengzhou University, Zhengzhou 450007, Henan Province, China
}

\begin{abstract}
[Abstract] Objective: To study the clinical effect of modified circumcision in the treatment of phimosis and excessive foreskin. Methods: 110 patients with overly long foreskin or phimosis were divided into experimental group and control group by random number table method, with 55 cases in each group. The experimental group was treated with modified circumcision, and the control group was treated with traditional manual cutting and suture. Then the clinical effects of the two treatments were compared. Results: Two different surgical procedures were performed. During the operation, the blood loss of the experimental group was less than that of the control group; the operation time and wound healing time were shorter than those of the control group; after the operation, the incidence of related complications in the experimental group was $14.55 \%$, which was significantly lower than For $25.45 \%$ of the control group, the clinical treatment effect was obvious, and the difference was statistically significant $(\mathrm{P}<0.05)$. Conclusion: The clinical effect of modified circumcision in the treatment of patients with phimosis and excessive foreskin is good. Compared with the traditional manual cutting and suture treatment, it not only has the advantages of simple operation and clinical application, but also benefits the rehabilitation of patients. Rehabilitation time reduces the incidence of postoperative complications. It is an ideal treatment plan for this type of disease in the current clinic, and it is worthy of popularization and application in general surgery and reproductive surgery.
\end{abstract}

Key words: Overly long foreskin; Phimosis; Modified circumcision; Clinical effect

Publication date: May, 2021; Publication online: 31 May, 2021

*Corresponding author: Jiechang Ju, Ju15136138403@163.com

In order to effectively reduce the risk of phimosis and overly long foreskin patients suffering from sexual transmission, urethral inflammation and infection, the most effective clinical way is to perform circumcision for them. With the further improvement of people's living standards and the widespread popularization of medical and health care knowledge, the number of such cases admitted to reproductive surgery has gradually increased, and the purpose of medical treatment is relatively simple. In order to realize the development of circumcision in the direction of minimally invasive and cosmetic, a lot of clinical research has been done, and certain improvements have been made to the operation, which is the modified circumcision involved in this research. In order to deeply explore the clinical application effect of modified circumcision in reproductive surgery, many medical experts have done a lot of clinical trial research. Academician Chen Peng ${ }^{[1]}$ combined 100 cases of phimosis and overly long foreskin in two groups of patients underwent full-thickness excision-scissor circumcision and modified commercial ring circumcision in clinical trials and found that full-thickness excision-scissor ring The resection treatment plan not only cannot well control the amount of intraoperative foreskin plate resection, but also has the risk of irregular margins and high postoperative complications. However, the improvement is the internal ring internal French minimally invasive surgery, which is easy to operate during the operation. The wound bleeding is less; there is no need for sutures and dressing 
changes after the operation, and the healing time of the patient's incision is shorter, which is worthy of widespread clinical application; Pan Weichang ${ }^{[2]}$ and others performed modified circumcision and traditional circumcision for exploration To determine the clinical application feasibility and value of modified circumcision in the treatment of patients with phimosis and excessive foreskin, they used a random lottery method to match the hospital admissions from August 2017 to July 2018. Pathological conditions, and the patients themselves and their family members are aware of, and support the study of 48 cases of phimosis and excessive foreskin patients who participated in the study were divided into observation group and control group, each with 24 cases. The former was treated with modified circumcision and the latter was treated. Traditional circumcision, through the comparison of the clinical effects of the two surgical procedures, it is found that the clinical effect of modified circumcision in the treatment of patients with phimosis and excessive prepuce is very significant. Patients in the observation group who underwent the modified circumcision treatment plan were better than the control group in terms of intraoperative blood loss, postoperative complications, and recovery time. Among them, the intraoperative blood loss from incisions in the observation group was $6.14 \pm 2.33 \mathrm{ml}$, which was significantly less than $13.76 \pm 2.67 \mathrm{ml}$ in the control group; postoperative complications in the observation group were $8.33 \%$, which was significantly lower than $33.32 \%$ in the control group; in terms of recovery time, The average recovery time of the observation group is 1 to 3 weeks, while the control group needs 3 to 4 weeks. The recovery speed has a clear advantage. Therefore, the surgical plan can be generalized. This study aims to explore the differences in clinical indicators between modified circumcision and traditional manual cutting and suture in the treatment of patients with phimosis and overlength prepuce, and to verify the clinical application value of modified circumcision. The specific research process and results are now reported as follows.

\section{Materials and methods}

\subsection{General information}

The research subjects selected 110 patients with overly long foreskin or phimosis admitted to our hospital from February 2018 to February 2019. The study has been approved by the ethics committee of our hospital ${ }^{[3]}$. Diagnosis and inclusion criteria: (1) Meet the symptoms of phimosis or true prepuce; (2) Exclude sexually transmitted diseases and other special reasons requiring circumcision; (3) Age 4 to 13 years old; (4) The patient's family members signed the informed consent for the study. Exclusion criteria: (1) The patient and his family did not know, or did not obtain the informed consent form signed by the patient's family; (2) Allergic to study medication and anesthetic drugs; (3) Under 4 years of age. 100 patients with genuine foreskin or phimosis were divided into experimental group and control group by random number table method, 55 cases in each group. There was no significant difference in general information between the two groups of patients $(\mathrm{P}>0.05)$, see Table 1 .

Table 1. Comparison of general information of patients in the 2 groups [ $\bar{x} \pm s, \mathrm{n}(\%)]$

\begin{tabular}{|c|c|c|c|c|c|}
\hline Group & $\begin{array}{l}\text { Number } \\
\text { of cases }\end{array}$ & $\begin{array}{c}\text { Average } \\
\text { age }\end{array}$ & $\begin{array}{c}\text { Average } \\
\text { weight }\end{array}$ & Inclusion criteria & Exclusion criteria \\
\hline Control group & 55 & $8.24 \pm 2.54$ & $24.9 \pm 4.90$ & $\begin{array}{l}\text { (1) Meet the symptoms of } \\
\text { phimosis or true prepuce; (2) } \\
\text { Exclude sexually transmitted } \\
\text { diseases and other special } \\
\text { reasons requiring circumcision; } \\
\text { (3) Age from } 4 \text { to } 13 \text { years old; } \\
\text { (4) The patient's family } \\
\text { members signed the informed } \\
\text { consent for the study }\end{array}$ & $\begin{array}{l}\text { (1) The patient himself } \\
\text { and his family members } \\
\text { did not know, or did not } \\
\text { obtain the informed } \\
\text { consent form signed by } \\
\text { the patient's family } \\
\text { members; (2) Allergic to } \\
\text { research medications and } \\
\text { anesthetics; (3) Under } 4 \\
\text { years of age }\end{array}$ \\
\hline
\end{tabular}




\subsection{Methods}

\subsubsection{Control group}

The 55 patients included in the control group were treated with traditional manual cutting and suture, and the specific operating procedures were strictly in accordance with the methods described in urology. Local cleansing, skin preparation, and dorsal penile nerve block anesthesia.

\subsubsection{Test group}

The 55 patients included in the trial were treated with the inner ring built-in method. Before the operation, the patient's penis size was accurately measured to select the appropriate type of circumcision device. During the operation, the patient was in a supine position, and after local routine disinfection, the anesthesia method was anesthesia with dorsal penile nerve block. After successful anesthesia, first use a hemostatic forceps to separate the adhering foreskin. After successful separation, place the inner ring into the coronal sulcus position. Note the length of the frenulum, and then put the circulator along the outer ring of the circulator. And remove the excess foreskin. After removing the excess foreskin, routinely disinfect the incision with iodophor, and take antibiotics orally for 3 to 5 days after the doctor's advice. 1 week after the operation, check the wound healing, consider removing the circumcision device, or wait for it to fall off by itself ${ }^{[4]}$.

\subsection{Observation indicators}

Intraoperative blood loss, operation time, postoperative wound healing time, incision swelling, rupture, and infection rates were compared between the two groups. Bleeding was calculated with $5 \mathrm{ml}$ per piece of gauze $(6 \mathrm{~cm} \times 8 \mathrm{~cm})$; postoperative edema was calculated with the ratio of the circumference of the foreskin inner plate at a distance of 10 $\mathrm{mm}$ from the coronary sulcus before and after the operation to exclude individual differences in the circumference of the penis ${ }^{[5]}$.

\subsection{Statistical methods}

Statistical analysis was performed with SPSS18.0 software. Measurement data is expressed by independent sample $t$ test for comparison between groups, paired sample $t$ test is used for comparison between groups, count data is expressed by rate, and comparison is expressed by $\chi 2$ test. $\mathrm{P}<0.05$ indicates that the difference is statistically significant.

\section{Results}

\subsection{Intraoperative index comparison}

Through comparison, the two groups of patients had significant differences in intraoperative blood loss, operation time, and incision healing time. The test group was better than the control group, and the difference was statistically significant $(\mathrm{P}<0.05)$, see Table 2 .

Table 2. Comparison of intraoperative blood loss, operation time and incision healing time in the two groups ( $\bar{x} \pm s$ )

\begin{tabular}{ccccc}
\hline Group & Number of cases & Intraoperative blood loss $(\mathbf{m l})$ & Operation time (min) & Incision healing time (d) \\
\hline Test group & 55 & $4.2 \pm 1.0$ & $20.5 \pm 1.5$ \\
Control group & 55 & $6.8 \pm 1.5$ & $26.1 \pm 1.5$ \\
\hline
\end{tabular}

\subsection{Comparison of clinical efficacy}

By comparing the incidence of postoperative incision swelling, rupture, infection and other related complications in the two groups of patients, the experimental group
$(14.55 \%)$ was significantly lower than the control group $(25.45 \%)$, and the difference was statistically significant $(\mathrm{P}<0.05)$. See Table 3 .

Table 3. Comparison of the incidence of postoperative complications in the two groups (n, \%)

\begin{tabular}{cccccc}
\hline Group & Number of cases & Incision swelling & Incision rupture & Incision infection & Complications \\
\hline Test group & 55 & $3(5.45)$ & $4(7.27)$ & $7(12.73)$ & $14(25.45)$ \\
Control group & 55 & $1(1.82)$ & $2(3.63)$ & $5(9.09)$ & $8(14.55)$ \\
\hline
\end{tabular}

\subsection{Safety evaluation}

During the treatment of the two groups of patients, no obvious drug side effects occurred.

\section{Discussion}

Phimosis or foreskin is too long to bring serious harm to the patient's health, and it can cause edema, congestion, erosion of the foreskin glans mucosa, long-term inflammation and stimulation, and even cancer of the penis, threatening the life of the patient ${ }^{[6]}$. At present, surgical treatment is often used 
in the treatment of phimosis or excessive foreskin.

In the past, due to the immature medical technology, manual cutting and suture were often used in the treatment of phimosis or overlength of the foreskin. This surgical plan not only failed to control the amount of resection of the inner and outer plates of the foreskin, but also had irregular cutting edges and The postoperative complications rate is high and the risk is high, but the improvement is that the internal ring is built-in French minimally invasive surgery, the operation is simple, the wound bleeding is less, the healing speed is fast, and the incidence of postoperative complications is low, which is the current clinical practice One of the most commonly used surgical options on the Internet $^{[7,8]}$.

Through this clinical trial study, it is shown that the modified circumcision has a very good effect in the treatment of phimosis and excessive foreskin. In the experimental group, patients in the experimental group have no matter the amount of bleeding during the operation, the time-consuming operation, or the healing time of the postoperative incision. The incidence of related complications was significantly lower than that of the control group $(\mathrm{P}<0.05)$, and it is worthy of clinical application.

\section{References}

[1] Chen P. Analysis of the clinical effect of modified Shanghuan circumcision in the treatment of excessive foreskin or phimosis in children[J]. China Continuing
Medical Education, 2016-(11)-10.

[2] Tang H, Gui B, Long WF. Observation on the use of modified circumcision in the treatment of phimosis and excessive foreskin $[\mathrm{J}]$. Chinese Community Physician, 2021-(02)-15.

[3] Zhang HF, Xia L. The clinical application of "four-step method" cuff-type modified surgery in outpatients with overly long prepuce $[\mathrm{J}]$. Chinese Journal of Andrology, 2020-(10)-20.

[4] Pan WC, Zheng SL. Comparison of the curative effect of modified circumcision and traditional circumcision in the treatment of excessive prepuce[J]. China Rural Health, 2019-(05)-28.

[5] Hou Q. Discussion on the method and effect of improved sleeve circumcision in the treatment of excessively long foreskin and phimosis[J]. Chinese and foreign medical care, 2016, (3): 77-78.

[6] Liu YY . Analysis of the clinical effects of different circumcision procedures in the treatment of excessive foreskin [J]. Chinese community physicians, 2017, (4): 26-27.

[7] $\mathrm{Gu} \mathrm{BH}$, Tian RH, Zhi EL, et la. Comparison of 73 cases of clinical efficacy between Shang ring circumcision and modified sleeve circumcision[J]. Chinese Journal of Andrology, 2017, (5): 53-56 .

[8] Li J, Zhu DC, Nie L. Comparison of the curative effect of modified sleeve circumcision and traditional circumcision[J]. China Medical Cosmetology, 2016, (3): $40-41$. 